\title{
Clinical outcomes in stage III non-small cell lung cancer patients treated with durvalumab after sequential or concurrent platinum- based chemoradiotherapy - single institute experience
}

\author{
Martina Vrankar ${ }^{1,2}$, Karmen Stanic ${ }^{1,2}$, Stasa Jelercic ${ }^{1}$, Eva Ciric ${ }^{1}$, Ana Lina Vodusek ${ }^{1}$ \\ Jasna But-Hadzic ${ }^{1,2}$ \\ ${ }^{1}$ Institute of Oncology Ljubljana, Department of Radiotherapy, Ljubljana, Slovenia \\ 2 University of Ljubljana, Faculty of Medicine, Ljubljana, Slovenia \\ Radiol Oncol 2021; 55(4): 482-490. \\ Received 19 August 2021 \\ Accepted 7 October 2021 \\ Correspondence to: Jasna But-Hadzic, M.D., Ph.D., Institute of Oncology Ljubljana, Zaloska 2, SI-1000 Ljubljana, Slovenia. \\ E-mail: jbut@onko-i.si; \\ Disclosure: No potential conflicts of interest were disclosed. \\ This is an open access article under the CC BY-NC-ND license (http://creativecommons.org/licenses/by-nc-nd/4.0/).
}

Background. Chemoradiotherapy (ChT-RT) followed by 12-month durvalumab is the new standard treatment for unresectable stage III non-small cell lung cancer. Survival data for patients from everyday routine clinical practice is scarce, as well as potential impact on treatment efficacy of sequential or concomitant chemotherapy and the usage of gemcitabine.

Patients and methods. We retrospectively analysed unresectable stage III NSCLC patients who were treated with durvalumab after radical concurrent or sequential chemotherapy (ChT) from December 2017 and completed treatment until December 2020. We assessed progression free survival (PFS), overall survival (OS) and toxicity regarding baseline characteristic of patients.

Results. Eighty-five patients with median age of 63 years of which $70.6 \%$ were male, $56.5 \%$ in stage IIIB and $58.8 \%$ with squamous cell carcinoma, were included in the analysis. Thirty-one patients received sequential ChT only, 51 patients received induction and concurrent $\mathrm{ChT}$ and 3 patients received concurrent ChT only. Seventy-nine patients (92.9\%) received gemcitabine and cisplatin as induction chemotherapy and switched to etoposide and cisplatin during concurrent treatment with radiotherapy (RT). Patients started durvalumab after a median of 57 days (range 12-99 days) from the end of the RT and were treated with the median of 10.8 (range 0.5-12 months) months. Forty-one patients (48.2\%) completed treatment with planned 12-month therapy, 25 patients (29.4\%) completed treatment early due to the toxicity and 16 patients (18.8\%) due to the disease progression. Median PFS was 22.0 months, 12-and estimated 24-month PFS were $71 \%$ (95\% Cl: 61.2-80.8\%) and $45.8 \%$ (95\% Cl: 32.7-58.9\%). With the median follow-up time of 23 months (range 2-35 months), median OS has not been reached. Twelve- and estimated 24-month OS were $86.7 \%$ (95\% Cl: $79.5-93.9 \%)$ and $68.6 \%$ (95\% Cl: $57.2-79.9 \%)$.

Conclusions. Our survival data are comparable with published research as well as with recently published real-world reports. Additionally, the regimen with gemcitabine and platinum-based chemotherapy as induction treatment was efficient and well tolerated.

Key words: non-small cell lung cancer; stage III; chemoradiotherapy; durvalumab 


\section{Introduction}

In the last few years, standard treatment of unresectable stage III non-small cell lung cancer (NSCLC) changed considerably after the publication of improved survival results with maintenance 12-month treatment with Programmed Death Ligand 1 (PD-L1) antibody durvalumab following standard concurrent chemoradiotherapy (ChTRT) ${ }^{1-3}$ In the PACIFIC trial, the median progression-free survival (PFS) from randomization was 17.2 months in durvalumab arm versus 5.6 months in placebo arm, while median overall survival (OS) was 47.5 months vs. 29.1 months, respectively. Reported 48 -month OS rates were $49.6 \%$ for durvalumab vs. $36.3 \%$ for placebo. ${ }^{4}$ However, survival data for stage III NSCLC patients treated with durvalumab after ChT-RT in every day routine clinical practice is scarce, as well as the survival data of patients treated with sequential ChT-RT followed by durvalumab. Patients with unresectable stage III NSCLC are highly heterogeneous regarding age, performance status (PS) and comorbidity and high proportion of them are not fit for concurrent ChT-RT. ${ }^{5,6}$ Here we present single centre survival and safety results for the treatment of unresectable stage III NSCLC patients with sequential or concurrent ChT-RT and maintenance durvalumab.

\section{Patients and methods}

\section{Patients and treatment}

We retrospectively analysed unresectable stage III NSCLC patients (according to the 8th TNM classification) who were considered for maintenance treatment with durvalumab (intention to treat population, ITT) after radical ChT-RT and completed treatment until December 2020.7 First 61 patients were included in early access program (EAP) which started in December 2017 and ended in September 2019 when reimbursement was introduced. Afterwards patients were treated with durvalumab as a standard of care. During EAP, patients were treated with durvalumab after at least stable disease with ChT-RT was achieved regardless of PD-L1 expression level, but from September 2019, during standard of care treatment, only patients with PD-L1 $\geq 1 \%$ received adjuvant durvalumab according to European Medicines Agency (EMA) registration.

Before treatment, patients underwent a physical examination, computed tomography (CT) of the chest, abdomen and head as well as the
${ }^{18} \mathrm{~F}$-fluorodeoxyglucose positron emission tomography/computed tomography $\left({ }^{18} \mathrm{~F}-\mathrm{FDG}\right.$ PET/CT) and brain magnetic resonance imaging (MRI) when indicated. All patients had histologically or cytologically confirmed NSCLC from primary tumour or regional lymph nodes, N stage mostly confirmed with endobronchial ultrasound (EBUS). PD-L1 immunohistochemistry was evaluated with a rabbit monoclonal antibody SP263 as part of the Ventana PD-L1 SP263 assay (Ventana/Roche, USA) on an automated platform (Benchmark, Ventana/ Roche, USA). According to our institutional clinical practice most patients started treatment with platinum based ChT combined with gemcitabine or pemetrexed and continued with platinum based ChT with the addition of etoposide or pemetrexed concurrently with RT. The prescribed radiation dose ranged from 54 Gray (Gy) to 66 Gy in 2 Gy daily fractions. Treatment was planned with ThreeDimensional Conformal Radiotherapy (3D-CRT) or Volumetric Modulated Arc Therapy (VMAT) with four-dimensional CT (4D CT) simulation in case of extensive target motion. Daily cone-beam CT was used for set-up correction. After completion of ChT-RT patients were evaluated with CT scan of the chest and abdomen, and when indicated, CT of the brain. Patients without progression and with resolved toxic effects of previous treatment started durvalumab within 3 months after ChT-RT for 12 months until progression or until unacceptable toxicity. Evaluation thoracic CT was done 6 and 12 months after durvalumab introduction and when clinically indicated.

\section{Statistical analysis}

Baseline characteristics of patients, including age, gender, pathological features, TNM stage, Eastern Cooperative Oncology Group performance status (ECOG PS), smoking status, PD-L1 expression, mutational status of EGFR, KRAS, ALK, ROS 1, BRAF and NTRK in adenocarcinoma, time to durvalumab start from the end of the RT, treatment completion, PFS and OS from the start of ChT-RT and the start of durvalumab were collected for the analysis. Response rate after ChT-RT was assessed using RECIST 1.1 and during immunotherapy iRECIST. ${ }^{8}$ Immune related adverse events were assessed by their highest reported grade using Common Terminology Criteria for Adverse Events (CTCAE) Version 5.0. ${ }^{9}$

PFS was calculated from the beginning of the durvalumab to disease progression or death and OS as the time from the start of the durvalumab to 
TABLE 1. Baseline characteristics of patients treated with durvalumab

\begin{tabular}{|c|c|c|}
\hline & & $N=85$ \\
\hline \multirow{2}{*}{ Gender } & Female & $25(29.4 \%)$ \\
\hline & Male & $60(70.6 \%)$ \\
\hline \multirow{3}{*}{ Age } & Median (range) & \\
\hline & $<63$ & $63(36-73)$ \\
\hline & $\geq 63$ & \\
\hline \multirow{3}{*}{ ECOG PS } & 0 & $37(43.5 \%)$ \\
\hline & 1 & $46(54.1 \%)$ \\
\hline & 2 & $2(2.4 \%)$ \\
\hline \multirow{3}{*}{ Smoking history } & Never & $2(2.4 \%)$ \\
\hline & Ex-smokers & $35(41.6 \%)$ \\
\hline & Smoking at diagnosis & $47(56.0 \%)$ \\
\hline \multirow{3}{*}{ Histology } & Adenocarcinoma & $31(36.5 \%)$ \\
\hline & Squamous Cell & $50(58.8 \%)$ \\
\hline & Other & $4(4.7 \%)$ \\
\hline \multirow{3}{*}{ AJCC 8th Edition Stage } & IIIA & $26(30.6 \%)$ \\
\hline & IIIB & $48(56.5 \%)$ \\
\hline & IIIC & $11(12.9 \%)$ \\
\hline \multirow{4}{*}{ PD-L1 Expression } & $<1 \%$ & $13(15.3 \%)$ \\
\hline & $1 \%-49 \%$ & $33(38.8 \%)$ \\
\hline & $>50 \%$ & $32(37.7 \%)$ \\
\hline & Unavailable & $7(8.2 \%)$ \\
\hline \multirow{3}{*}{ Mutational status } & No mutations & $65(76.5 \%)$ \\
\hline & KRAS & $16(18.8 \%)$ \\
\hline & Unavailable & 4 (4.7%) \\
\hline
\end{tabular}

Abbreviation: N-number, ECOG PS- Eastern Cooperative Oncology Group performance status, PD-L1-programmed dead-ligand 1
This study was conducted in accordance with the Declaration of Helsinki. The study was approved by Institutional Review Board Committee and Institutional Ethics Committee (ERIDNPVO-0004/2021).

\section{Results}

In total, 118 patients had been identified as candidates for maintenance treatment with durvalumab from December 2017 who completed treatment until December 2020. Of those, 85 (72.0\%) patients continued treatment with maintenance durvalumab after ChT-RT and in 33 durvalumab was omitted due to persistent toxicity in 6 , treatment refusal in 11 and progressive disease in 16 patients.

Baseline characteristics of 85 patients included in the analyses are detailed in Table 1 . Most were male $(70.6 \%)$ in stage IIIB $(56.5 \%)$ with squamous cell carcinoma (58.8\%), ECOG PS 1 (54.1\%). Median age was 63 years (range $36-73$ years). PD-L1 expression was positive in $65(76.5 \%)$ patients, in 13 (15.3\%) patients was negative and not available in 7 patients (8.2\%). No EGFR, ALK, ROS 1, BRAF and TNRK mutations were detected in 65 (76.5\%) patients, KRAS mutation was present in $16(18.8 \%)$ patients and for 4 patients mutational status was not available.

\section{Treatment with chemoradiotherapy}

Eighty-two (96.5\%) patients started treatment with induction ChT and 54 (63.5\%) patients received ChT during RT (Table 2). Thirty-one (36.5\%) patients were treated with sequential ChT only. Patients received median 3 cycles (range 1-5) of ChT altogether. Seventy-nine patients $(92.9 \%)$ received gemcitabine and cisplatin as induction ChT and $52(61.2 \%)$ of those switched to etoposide and cisplatin during concurrent treatment with RT. Only 3 patients $(3.5 \%)$ with adenocarcinoma were treated with pemetrexed and cisplatin as induction as well as concurrent regimen. Patients were treated with the median RT dose of $60 \mathrm{~Gy}$ (range 54 Gy-66 Gy), and in most of them (82.3\%) partial response was observed after ChT-RT.

\section{Treatment with durvalumab}

Patients started first cycle of durvalumab after a median of 57 days (range 12-99 days) from the end of the RT and were treated with the median of 10.8 months (range 0.5-12 months) (Table 3). Forty-one 


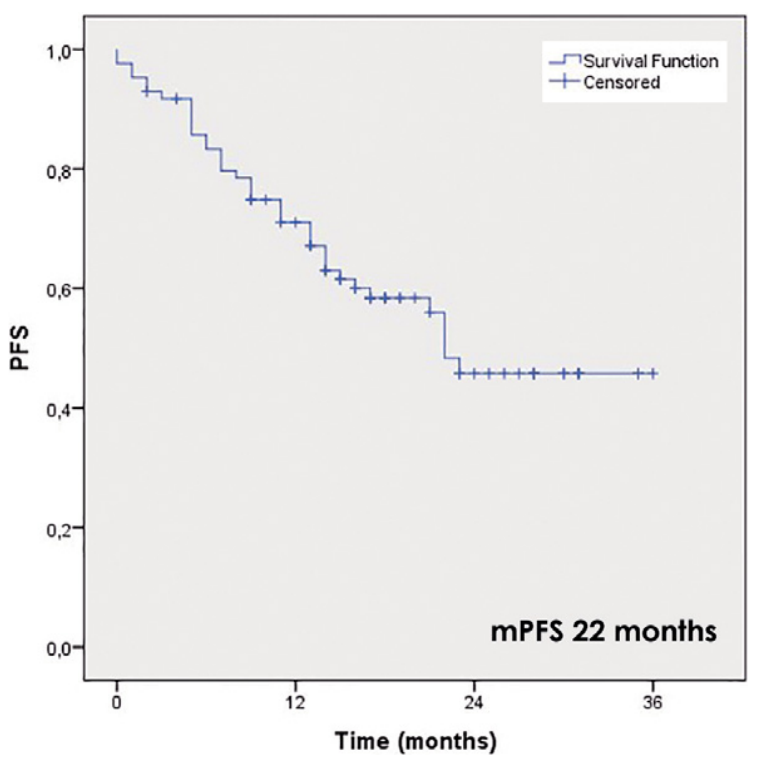

FIGURE 1. Progression free survival of patients treated with durvalumab after sequential or concurrent platinum-based chemoradiotherapy.

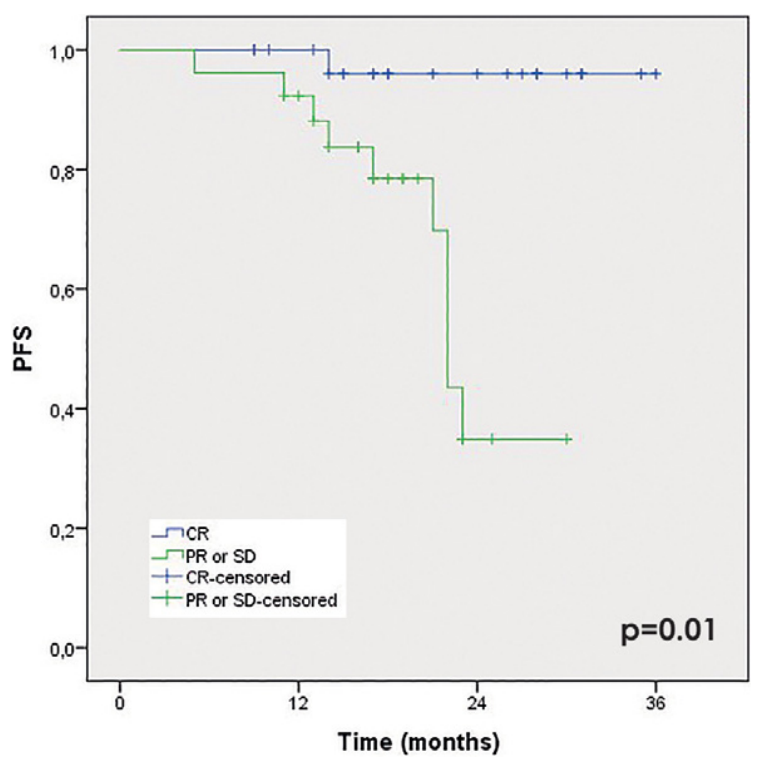

FIGURE 2. Progression free survival regarding response after durvalumab completion.

patients (48.2\%) completed treatment with planned 12 -month therapy, 25 patients $(29.4 \%)$ completed treatment early due to the toxicity of durvalumab and 16 patients (18.8\%) due to the disease progression. One patient died suddenly after two months of treatment without progression and no known cause of death. One patient stopped treatment after
TABLE 2. Chemoradiotherapy treatment characteristics

\begin{tabular}{|c|c|c|}
\hline & & $\mathrm{N}=85(\%)$ \\
\hline \multirow{5}{*}{$\mathrm{N}$ of $\mathrm{ChT}$} & 1 & $3(3.5 \%)$ \\
\hline & 2 & $13(15.3 \%)$ \\
\hline & 3 & $41(48.2 \%)$ \\
\hline & 4 & $27(31.8 \%)$ \\
\hline & 5 & $1(1.2 \%)$ \\
\hline \multirow{3}{*}{ ChT } & Gem/cis & 79 (92.9\%) \\
\hline & Etop/cis & $52(61.2 \%)$ \\
\hline & Pem/cis & $3(3.5 \%)$ \\
\hline \multirow{4}{*}{ ChT } & Induction & $82(96.5 \%)$ \\
\hline & Sequential only & 31 (36.5\%) \\
\hline & Concurrent & $54(63.5 \%)$ \\
\hline & Concurrent only & $3(3.5 \%)$ \\
\hline RT dose (Gy) & Median (range) & $60(5-66)$ \\
\hline V20 (Gy) & Median (range) & $27.2(7.0-35.6)$ \\
\hline MLD (Gy) & Median (range) & $15.7(4.0-20.2)$ \\
\hline PTV $\left(\mathrm{cm}^{3}\right)$ & Median (range) & $416.6(172.3-1282.6)$ \\
\hline \multirow{3}{*}{ Evaluation after ChT-RT } & $\mathrm{CR}$ & 10 (11.8\%) \\
\hline & PR & 70 (82.3\%) \\
\hline & SD & $5(5.9 \%)$ \\
\hline $\begin{array}{c}\text { Time between RT-IT } \\
\text { (days) }\end{array}$ & Median (range) & 57 (12-99) \\
\hline
\end{tabular}

$\mathrm{ChT}=$ chemotherapy, $\mathrm{CR}=$ complete response; etop/cis = etoposide/cisplatin; $\mathrm{gem} / \mathrm{cis}=$ gimcitabine/cisplatin; IT = immunotherapy; $M L D=$ mean lung dose; $N=$ number of patients; $P D=$ progressive disease; PR = partial response; $\mathrm{PTV}=$ planning target volume; $\mathrm{RT}$ = radiotherapy; pem/ $\mathrm{cis}=$ pemetrexed/cisplatin; $\mathrm{SD}=$ stable disease; $\mathrm{V} 20$ = volume of the lung that receive radiation dose of $20 \mathrm{~Gy}$

one month due to newly diagnosed prostate cancer and one due to cerebral infarction unrelated to durvalumab treatment.

\section{Toxicity of durvalumab treatment}

Twenty-five patients $(29.4 \%)$ discontinued durvalumab early due to the toxicity after the median treatment time of 6.0 months (range 0.5-11 months). Twelve patients (14.1\%) had pneumonitis that started significantly earlier after introduction of durvalumab than other AE (2.0 months vs. 7.2 months, $p=0.012$ ). Other AE leading to discontinuation of durvalumab treatment included dermatological toxicity $(n=5)$, arthralgia $(n=4)$, colitis $(n=$ 2 ) and uncontrolled hypothyroidism $(n=2)$. Most immune related $\mathrm{AE}$ leading to discontinuation of durvalumab were grade $1-2(68 \%), 32 \%$ were grade 3. No grade 4 of $5 \mathrm{AE}$ were observed. 
TABLE 3. Durvalumab treatment characteristics and influence on overall survival

\begin{tabular}{|c|c|c|c|}
\hline \multicolumn{2}{|c|}{ Treatment characteristics } & N (\%) & $\mathbf{P}$ \\
\hline \multicolumn{4}{|c|}{ Median (days) } \\
\hline \multirow[t]{2}{*}{ Time between RT-IT } & $<57$ & 57 (12-99) & 0.689 \\
\hline & $\geq 57$ & & \\
\hline \multirow{3}{*}{ Treatment time of IT* } & Median (months) & & \\
\hline & $<10.8$ & $10.8(0.5-12.0)$ & $<0.001$ \\
\hline & $\geq 10.8$ & & \\
\hline \multirow{4}{*}{ Treatment with IT } & Completed & $41(48.2 \%)$ & \multirow{4}{*}{0.095} \\
\hline & Early stopped due to $\mathrm{AE}$ & $25(29.4 \%)$ & \\
\hline & Progression & $16(18.8 \%)$ & \\
\hline & Other & $3(3.6 \%)$ & \\
\hline \multirow{4}{*}{ Response after IT** } & CR & 29 (34.1\%) & \multirow{4}{*}{0.213} \\
\hline & PR & $11(12.9 \%)$ & \\
\hline & SD & $15(17.6 \%)$ & \\
\hline & PD & 10 (11.8\%) & \\
\hline \multirow{3}{*}{ Progression ${ }^{* * *}$} & Loco-regional & $21(24.7 \%)$ & \multirow{3}{*}{0.217} \\
\hline & Metastatic & $10(11.7 \%)$ & \\
\hline & Metastatic and local & $5(5.9 \%)$ & \\
\hline \multirow{2}{*}{ Metastatic spread } & CNS & $5(5.9 \%)$ & \multirow{2}{*}{0.101} \\
\hline & Other & $10(11.7 \%)$ & \\
\hline
\end{tabular}

*difference in overall survival between patients treated with immunotherapy less or more than median time;

** including evaluation up to 4 months after completed immunotherapy in patients with 12-month therapy as well as in early stopped due to adverse events, later progression is not included. Two patients were not evaluable:

*** observed progression until the last evaluation date:

$\mathrm{AE}=$ adverse events; $\mathrm{CNS}=$ central nerve system; $\mathrm{CR}=$ complete response; $\mathrm{IT}=$ immunotherapy; $\mathrm{PD}=$ progressive disease; $\mathrm{PR}=$ partial response; $\mathrm{RT}$ = radiotherapy; $\mathrm{SD}=$ stable disease

In total, 42 (49.6\%) patients experienced $47 \mathrm{AE}$, 38 patients had $1 \mathrm{AE}$, three patients had 2 and one patient had 3 AE. Pneumonitis was found in 15 $(17.6 \%)$, arthralgia in $5(5.9 \%)$, skin toxicity in 13 $(15.3 \%)$, colitis in $6(7.1 \%)$ and hypothyroidism in $8(9.4 \%)$ patients.

\section{Treatment results}

Median PFS from the durvalumab start was 22.0 months, estimated 12- and 24-month PFS were 71\% (95\% CI: $61.2-80.8 \%$ ) and $45.8 \%$ (95\% CI: $32.7-$ $58.9 \%$ ) (Figure 1). During durvalumab treatment, 16 patients progressed after the durvalumab treatment median time of 6.1 months (range 0.5-11.0 months), 7 with loco-regional and 9 with distant metastases. Altogether, $36(42.4 \%)$ patients have progressed until the last follow-up date, 21 (24.7\%) patients with loco-regional failure only and 15 $(17.6 \%)$ patients with distant metastatic disease. Of those, 5 patients had also local progression.

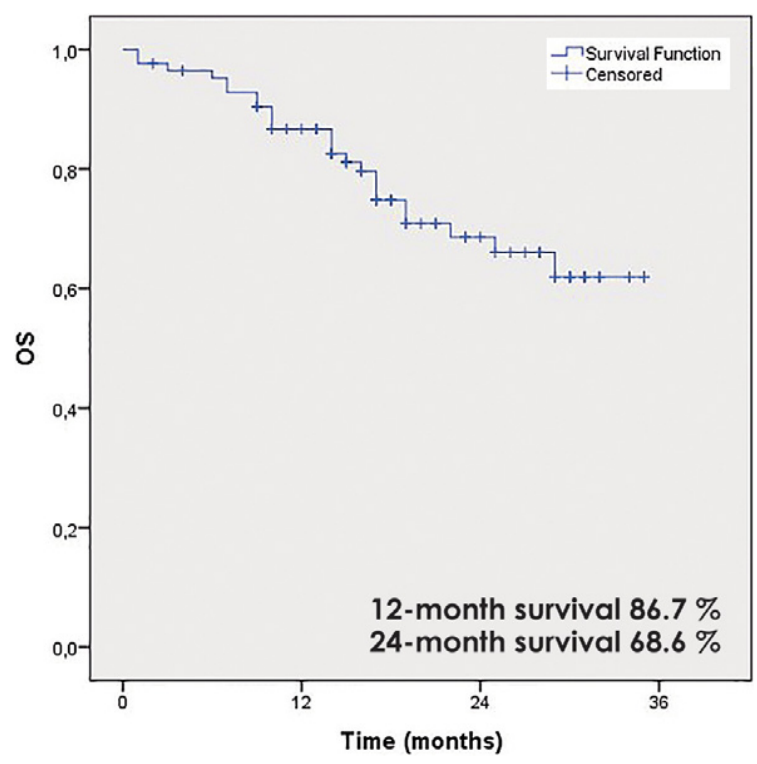

FIGURE 3. Overall survival of patients treated with durvalumab after sequential or concurrent platinum-based chemoradiotherapy.

Age, gender, ECOG PS, stage, histology, PD-L1 expression, mutational status, smoking status, RT dose, time between end of RT and start of durvalumab, time of durvalumab treatment in nonprogressive patients during durvalumab, ChT sequential vs. concurrent, did not predict poorer PFS in univariate analysis (Table 4). Patients who had complete response (CR) on CT evaluation after durvalumab treatment had significantly longer PFS compared to those with partial response (PR) or stable disease (SD) (mPFS not reached vs. 22 months, $\mathrm{p}=0.01$ ) and this was affirmed in multivariate analysis (Figure 2).

Regarding the pattern of progression, we found more loco-regional only progression in squamous cell carcinoma $(79.4 \%)$ and distant metastases in adenocarcinoma $(81.8 \%)$, the difference was significant $(p=0.002)$ (Table 5). In addition, all patients with KRAS mutation that progressed, had distant metastases only $(p<0.001)$. On the contrary, all patients that discontinued treatment early due to AE and progressed later $(n=8)$, had loco-regional failure only $(\mathrm{p}=0.024)$.

Median OS from the durvalumab start has not been reached after the median follow-up time of 23 months (range 2-35 months). Twelve- and estimated 24-month OS were $86.7 \%$ (95\% CI: 79.5-93.9\%), and $68.6 \%$ (95\% CI: 57.2-79.9\%), respectively (Figure 3). In total, 25 patients $(29.4 \%)$ have died until the last follow-up date. 
TABLE 4. Univariate and multivariate analysis of predictors for progression free survival

\begin{tabular}{|c|c|c|c|c|c|}
\hline \multicolumn{2}{|c|}{ Variable } & \multicolumn{2}{|c|}{ Univariate analysis } & \multicolumn{2}{|c|}{ Multivariate analysis } \\
\hline . & & HR $(95 \% \mathrm{Cl})$ & $p$ & HR $(95 \% \mathrm{Cl})$ & $p$ \\
\hline \multirow{2}{*}{ Age } & $<63$ years & $0.67(0.35-1.29)$ & 0.231 & & \\
\hline & $\geq 63$ years & & & & \\
\hline \multirow{2}{*}{ Gender } & Male & $0.62(0.32-1.20)$ & 0.156 & & \\
\hline & Female & & & & \\
\hline \multirow{2}{*}{ ECOG PS } & 0 & $0.66(0.34-1.28)$ & 0.226 & & \\
\hline & $1-2$ & & & & \\
\hline \multirow{3}{*}{ Stage } & IIIA & $0.89(0.54-1.48)$ & 0.663 & & \\
\hline & IIIB & & & & \\
\hline & IIIC & & & & \\
\hline \multirow{2}{*}{ Histology } & Adenocarcinoma & $0.84(0.41-1.70)$ & 0.635 & & \\
\hline & Squamous Cell & & & & \\
\hline \multirow{3}{*}{ Smoking status } & Never & $0.59(0.33-1.07)$ & 0.084 & $1.72(0.26-11.00)$ & 0.567 \\
\hline & Ex-smokers & & & & \\
\hline & Smoking at diagnosis & & & & \\
\hline \multirow{2}{*}{ Mutational status } & No & $0.99(0.87-1.12)$ & 0.881 & & \\
\hline & KRAS & & & & \\
\hline \multirow{3}{*}{ PD-L1 } & $<1 \%$ & $1.03(0.67-1.59)$ & 0.882 & & \\
\hline & $1 \%-49 \%$ & & & & \\
\hline & $>50 \%$ & & & & \\
\hline \multirow{2}{*}{ Time to durvalumab } & $<57$ days & $0.63(0.32-1.24)$ & 0.186 & & \\
\hline & $\geq 57$ days & & & & \\
\hline \multirow{2}{*}{ RT dose } & $<60$ Gy & $1.10(0.43-2.84)$ & 0.838 & & \\
\hline & $\geq 60 \mathrm{~Gy}$ & & & & \\
\hline \multirow{2}{*}{ No of ChT } & Up to 3 & $2.06(0.94-4.51)$ & 0.069 & $0.83(0.23-2.99)$ & 0.783 \\
\hline & $4-5$ & & & & \\
\hline \multirow{2}{*}{ ChT } & Sequential & $1.51(0.79-2.85)$ & 0.209 & & \\
\hline & Concurrent & & & & \\
\hline \multirow{2}{*}{ Response after IT } & CR & $0.066(0.008-0.518)$ & 0.010 & $0.067(0.008-0.535)$ & 0.011 \\
\hline & $\mathrm{PR} / \mathrm{SD}$ & & & & \\
\hline \multirow{2}{*}{$\begin{array}{l}\text { Durvalumab treatment } \\
\text { time }\end{array}$} & $<10.8$ months & $3.16(1.62-6.17)$ & 0.001 & $1.18(0.227-6.17$ & 0.841 \\
\hline & $\geq 10.8$ months & & & & \\
\hline
\end{tabular}

$\mathrm{CR}=$ complete response; $\mathrm{ECOG}$ PS = Eastern Cooperative Oncology Group performance status; $\mathrm{ChT}=\mathrm{chemotherapy}, \mathrm{RT}=$ radiotherapy, $\mathrm{IT}=$ immunotherapy; $\mathrm{PD}=$ progressive disease; $\mathrm{PD}-\mathrm{LI}$ = programmed dead-ligand $1 ; \mathrm{PR}=$ partial response; $\mathrm{SD}=$ stable disease

Age, gender, ECOG PS, stage, histology, PD-L1 expression, mutational status, smoking status, RT dose, ChT sequential vs. concurrent, the time between end of RT and start of durvalumab did not predict poorer OS in univariate analysis. Patients with CR after ChT-RT had significant longer survival with Kaplan-Meier method than patients with PR or SD $(p=0.045)$, however, survival was not different according to response after dur- valumab treatment. In the multivariate Cox proportional hazards model CR after ChT-RT as well as after completion of durvalumab was not predictor of better OS.

\section{Treatment after progression}

After progression, six patients had no additional treatment, mostly due to progressive deterioration 
TABLE 5. Pattern of progression

\begin{tabular}{|c|c|c|c|c|}
\hline \multicolumn{2}{|c|}{ Progression ( $\mathrm{N}$ of patients) } & \multirow{2}{*}{$\begin{array}{c}\begin{array}{c}\text { Loco-regional } \\
\text { only }\end{array} \\
12\end{array}$} & \multirow{2}{*}{$\begin{array}{c}\text { Metastatic } \\
10\end{array}$} & \multirow{3}{*}{$\begin{array}{c}\mathbf{p} \\
0.563\end{array}$} \\
\hline$\rho_{2}$ & Male & & & \\
\hline Sertuc & Female & 9 & 5 & \\
\hline \multirow{3}{*}{ Stage } & IIIA & 7 & 3 & \multirow{3}{*}{0.663} \\
\hline & $\| I I B$ & 11 & 9 & \\
\hline & IIIC & 3 & 3 & \\
\hline \multirow{3}{*}{ Histology } & Adenocarcinoma & 2 & 9 & \multirow{3}{*}{0.002} \\
\hline & Squamous cell & 19 & 5 & \\
\hline & Other & 0 & 1 & \\
\hline \multirow{2}{*}{ Mutation } & KRAS & 0 & 7 & \multirow{2}{*}{$<0.001$} \\
\hline & No mutation & 21 & 8 & \\
\hline \multirow{3}{*}{ PD-L 1} & $<1 \%$ & 3 & 4 & \multirow{3}{*}{0.435} \\
\hline & $1 \%-49 \%$ & 8 & 6 & \\
\hline & $50 \%$ & 10 & 4 & \\
\hline
\end{tabular}

$\mathrm{N}=$ number; PD-L1 = programmed dead-ligand 1

of patient's performance. Fourteen patients were treated with RT only and eleven with ChT, of those six received aditional $\mathrm{RT}$ and one of them also immunotherapy. Two patients had surgery of brain metastases and three patients had salvage surgery of primary tumour.

\section{Discussion}

The results of PFS and OS in our series of patients with stage III NSCLC treated with durvalumab after ChT-RT confirmed improved survival compared to our historical data of treatment before durvalumab introduction. ${ }^{10-12}$ After the median follow-up time of 23 months, 12- and 24-month PFS (71\% and $45.8 \%$, respectively) and OS (86.7\% and $68.6 \%$, respectively) in our series were comparable with those in the PACIFIC trial (PFS 55.3\% and $44.8 \%$ and OS $83.1 \%$ and $66.3 \%$ ). ${ }^{1-4}$ In addition, data from other real-world reports have confirmed the advantage of maintenance treatment with durvalumab over ChT-RT only. ${ }^{13-16}$ In a series of 62 patients in the report of Offin et al., the 12-month PFS and OS were $65 \%$ and $85 \%$, respectively. ${ }^{14}$ In the Canadian multicentre analysis with 147 patients included, 12-month OS rate of $92.5 \%$ was reported after a median follow-up of 15.8 months. ${ }^{15}$ Report from the German group encompassed 56 centres with altogether 126 patients treated in expanded access programme, revealed the 12- and 24-month
PFS of $56.0 \%$ and $46.7 \%$, and $12-$ and $24-$ month OS of $78.6 \%$ and $66.0 \%$, respectively. ${ }^{16}$

The broad usage of induction ChT before RT, and ChT selection of gemcitabine in our group of stage III NSCLC patients offered comparable survival rates and safety as reported with other schedules in recent publications. ${ }^{14-16}$ Some patients with stage III NSCLC are not candidates for concurrent ChT-RT due to the age and comorbidity. ${ }^{5,6}$ Most patients $(96.5 \%)$ in our series started treatment with ChT, majority of them with platinum-based ChT including gemcitabine and $63.5 \%$ of all continued platinum-based ChT during RT. In the PACIFIC study, only $25.8 \%$ of patients were treated with induction ChT and $99.8 \%$ of patients with concurrent platinum-based ChT with etoposide, vinblastine, vinorelbine, taxans or pemetrexed.1,17 Only few patients in PACIFIC trial were treated with gemcitabine, and additionally, gemcitabine was not used in none of the recently published real-world durvalumab treatment reports. In the real-world reports of durvalumab treatment, platinum-based ChT with etoposide was used in $11.0 \%$ to $21.8 \%$ of all patients concurrent with RT, and induction ChT was used in up to $32.5 \%$ of patients. ${ }^{14-16}$

Treatment with induction gemcitabine in our historical analysis had not revealed excessive AE ${ }^{10-}$ ${ }^{12}$. Induction, sequential and concomitant regimes were well tolerated also in the present series (data not shown). In present analysis, there were no differences in PFS and OS between the patients treated with sequential ChT only or concurrent ChT. Additionally, the effectiveness of platinum-based ChT with induction gemcitabine was not inferior to other ChT schedules when comparing PFS and OS. Comparing the best response to ChT-RT, we observed a higher rate of CR and PR (11.8\% and $82.3 \%$ ) than the PACIFIC trial $(1.9 \%$ and $48.7 \%) .{ }^{1-4}$ The median time to first cycle of durvalumab from the end of RT in our series was 57 days (range 12-99 days) which is considerably longer than in PACIFIC trial (range 1-42 days), and longer as reported by Offin with a median time of 1.5 months (range 0.3-7.7 months), and Desilets with 33 days (range 1-94 days). ${ }^{14,15}$ Early completed durvalum$\mathrm{ab}$ treatment due to progression in our analysis was observed in $18.8 \%$ of patients as compared to $30.2 \%$ in PACIFIC trial and $34.1 \%$ reported by Faehling. ${ }^{1,16}$ At the evaluation up to 4 months after the completion of durvalumab treatment (planned 12-month therapy or early completed due to AE), we observed CR, PR and SD in 34.1\%, $12.9 \%$ and $17.6 \%$ of patients. Notably, the only predictor for improved PFS in our series was CR compared to 
PR/SD after durvalumab treatment that was confirmed in multivariate analysis $(p=0.032)$.

Baseline characteristics of patients in our series differed from patients in PACIFIC trial. In our analysis, more patients in stage IIIB and IIIC $(69.4 \%$ vs. $47.0 \%)$ were included and more patients had squamous cell carcinoma ( $58.1 \%$ vs. $47.1 \%$ ). Both characteristics are known predictors for worse prognosis. ${ }^{18,19}$ Two real-world analysis similarly reported $72 \%$ and $68.3 \%$ of patients included in stage IIIB and IIIC NSCLC, but lower proportion of squamous cell carcinoma, ranged from $31 \%$ to $42.6 \% .{ }^{14,16}$ Squamous cell lung carcinoma is associated with inferior OS in all stage groups including unresectable stage III NSCLC. ${ }^{20}$ Some reports indicated that patients with squamous-cell carcinoma typically presented with bulky locally advanced disease and in those patients, it might be an advantage to start treatment with induction $\mathrm{ChT}$ as it was the case in our series. ${ }^{16}$ In our analysis, no difference in PFS and OS regarding histology was revealed, but significantly more loco-regional progression was observed compared to metastatic progression in squamous cell carcinoma $(\mathrm{p}=0.002)$ as in adenocarcinoma. Altogether, $24.7 \%$ of patients had loco-regional progression only and $17.6 \%$ had progression with metastatic disease. On the contrary, Offin et al. reported the 12-month incidence of loco-regional and distant failures of $18 \%$ and $30 \%{ }^{14}$ High proportion of loco-regional progression in our series might be due to high proportion of squamous cell carcinoma.

The salvage treatment for most patients with loco-regional progression was reirradiation with or without reinduction ChT. Three patients in our series had salvage surgery due to progressive primary tumour with observed regression of the lymph nodes. In all, the histology revealed down-staging of the lymph nodes and persistent malignant cells in the primary tumour. The high proportion of loco-regional failures only opens the important emerging issue how to deal with the patients after completion of ChT-RT and maintenance durvalumab with PR or SD. Regarding our results, surgery might be an appropriate additional treatment option in selected patients with PR, especially in patients with squamous cell carcinoma. Further clinical trials are investigating incorporation of immunotherapy at different time point in treatment with ChT-RT in stage III NSCLC patients. Additionally, the research in modulating the immune response by interfering with specific alternative immune receptors, pathways and mediators is ongoing and might offer additional knowledge that would affect the treatment of stage III NSCLC patients. ${ }^{21}$ However, as demonstrated in advanced NSCLC, one treatment might not be suitable for all and in the future, it could be revealed that personalized multimodality approach for selected stage III NSCLC patients might enable better survival results.

Our results presented here were collected as a single institution experience. Due to small number of patients, this series might be underpowered to detect significant impact on survival for different treatment regimens and probable prognostic variable. Also, some information in statistical analysis might be lost, due to dichotomisation of continuous data. Due to retrospective nature of the analysis, some data were not available for all patients. However, despite more advanced stage III NSCLC and squamous cell histology, our results are consistent with the PACIFIC trial. Additionally, further studies are warranted assessing management of patients with loco-regional SD or PR after durvalumab treatment.

\section{Conclusions}

The survival data in present analysis confirmed the advantage of maintenance durvalumab in the treatment of unresectable stage III NSCLC patients over ChT-RT only and our results are in line with the PACIFIC trial as well as with recently published real-world reports. Additionally, with mostly gemcitabine as induction platinum-based ChT, the survival outcomes confirmed our treatment regimen as efficient and well tolerated.

\section{Acknowledgments}

We thank all the patients who participated in this study.

\section{References}

1. Antonia SJ, Villegas A, Daniel D, Vicente D, Murakami S, Hui R, et al. Durvalumab after chemoradiotherapy in stage III Non-Small-Cell Lung Cancer. N Engl J Med 2017; 377: 1919-29. doi: 10.1056/NEJMoa1709937

2. Antonia SJ, Villegas A, Daniel D, Vicente D, Murakami S, Hui R, et al. Overall survival with durvalumab after chemoradiotherapy in stage III NSCLC. N Engl J Med 2018; 379: 2342-50. doi: 10.1056/NEJMoa1809697

3. Hui R, Ozguroglu M, Villegas A, Daniel D, Vicente D, Murakami S, et al. Patient-reported outcomes with durvalumab after chemoradiotherapy in stage III, unresectable non-small cell lung cancer (PACIFIC): a randomised, controlled, phase 3 study. Lancet Oncol 2019; 20: 1670-80. doi: 10.1016/ S1470-2045(19)30519-4 
4. Faivre-Finn C, Vicente D, Kurata T, Planchard D, Paz-Ares L, Vansteenkiste JH et al. Four-year survival with durvalumab after chemoradiotherapy in stage III NSCLC-an update from the PACIFIC Trial. J Thorac Oncol 2021; 16: 860-7. doi: 10.1016/j.jtho.2020.12.015

5. Yusuf D, Walton RN, Hurry M, Farrer D, Bebb DG, Cheung WY, et al. Population-based treatment patterns and outcomes for stage III non-small cell lung cancer patients. Am J Clin Oncol 2020; 43: 615-20. doi: 10.1097/ COC.0000000000000716

6. Yoon SM, Shaikh T, Hallman M. Therapeutic management options for stage III non-small cell lung cancer. World J Clin Oncol 2017; 8: 1-20. doi: 10.5306/ wjco.v8.i1.1

7. Brierly DJ, Gospodarowicz MK, Witekind C, editors. TNM classification of malignant tumours. 8th edition. Oxford: Wiley-Blackwell; 2017. p. 114-20.

8. Seymour L, Bogaerts J, Perrone A, Ford R, Schwartz LH, Mandrekar S, et al. iRECIST: guidelines for response criteria for use in trials testing immunotherapeutics. Lancet Oncol 2017; 18: e143-52. doi: 10.1016/S14702045(17)30074-8

9. NIH. National Cancer Institute. DCTD Division of Cancer Treatment \& Diagnosis. Common Terminology Criteria for Adverse Events (CTCAE). Version 5.O. U.S. Department of Health and Human Services; 2017. [cited $2021 \mathrm{Jul}$ 07]. Available at: https://ctep.cancer.gov/protocoldevelopment/ electronic_applications/docs/ctcae_v5_quick_reference_5x7.pdf

10. Vrankar M, Zwitter M, Bavcar T, Milic A, Kovac V. Induction gemcitabine in standard dose or prolonged low-dose with cisplatin followed by concurrent radiochemotherapy in locally advanced non-small cell lung cancer: a randomized phase II clinical trial. Radiol Oncol 2014; 48: 369-80. doi: 10.2478/ raon-2014-0026

11. Vrankar M, Stanic K. Long-term survival of locally advanced stage III nonsmall cell lung cancer patients treated with chemoradiotherapy and perspectives for the treatment with immunotherapy. Radiol Oncol 2018; 52: 281-8. doi: 10.2478/raon-2018-0009

12. Vrankar M, Kern I, Stanic K. Prognostic value of PD-L1 expression in patients with unresectable stage III non-small cell lung cancer treated with chemoradiotherapy. Radiat Oncol 2020; 15: 247. doi: 10.1186/s13014-020-01696-z

13. Taugner J, Käsmann L, Eze C, Tufman A, Reinmuth N, Duell T, et al Durvalumab after chemoradiotherapy for PD-L1 expressing inoperable stage III NSCLC leads to significant improvement of local-regional control and overall survival in the real-world setting. Cancers 2021; 13: 1613. doi: $10.3390 /$ cancers 13071613

14. Offin M, Shaverdian N, Rimner A, Lobaugh S, Shepherd AF, Simone CB 2nd et al. Clinical outcomes, local-regional control and the role for metastasisdirected therapies in stage III non-small cell lung cancers treated with chemoradiation and durvalumab. Radiother Oncol 2020; 149: 205-11. doi: 10.1016/j.radonc.2020.04.047

15. Desilets A, Blanc-Durand F, Lau S, Hakozaki T, Kitadai R, Malo J, et al. Durvalumab therapy following chemoradiation compared with a historical cohort treated with chemoradiation alone in patients with stage III nonsmall cell lung cancer: A real-world multicentre study. Eur J Cancer 2021; 142: 83-91. doi: 10.1016/j.ejca.2020.10.008

16. Faehling $M$, Schumann $C$, Christopoulos $P$, Hoffknecht $P$, Alt J, Horn M, et al. Durvalumab after definitive chemoradiotherapy in locally advanced unresectable non-small cell lung cancer (NSCLC): real-world data on survival and safety from the German expanded-access program (EAP). Lung Cancer 2020; 150: 114-22. doi: 10.1016/j.lungcan.2020.10.006

17. Faivre-Finn C, Spigel DR, Senan S, Langer C, Perez BA, Özgüroğlu M, et al. Impact of prior chemoradiotherapy-related variables on outcomes with durvalumab on unresectable Stage III NSCLC (PACIFIC). Lung Cancer 2021; 151: 30-8. doi: 10.1016/j.lungcan.2020.11.024

18. Jin G, Wang X, Xu C, Sun J, Yuan Z, Wang J, et al. Disparities in survival following surgery among patients with different histological types of N2-II non-small cell lung cancer: a Surveillance, Epidemiology and End Results (SEER) database analysis. Ann Trans/ Med 2020; 8: 1288. doi: 10.21037/ atm-20-4357

19. Morgensztern D, Waqar S, Subramanian J, Gao F, Trinkaus K, Govindan R, et al. Prognostic significance of tumor size in patients with stage III nonsmall-cell lung cancer: a surveillance, epidemiology, and end results (SEER) survey from 1998 to 2003. J Thorac Oncol 2012; 7: 1479-84. doi: https://doi. org/10.1097/JTO.0b013e318267d032
20. Wang BY, Huang JY, Chen HC, Lin $\mathrm{CH}$, Lin SH, Hung WH, et al. The comparison between adenocarcinoma and squamous cell carcinoma in lung cancer patients. J Cancer Res Clin Oncol 2020; 146: 43-52. doi: 10.1007/ s00432-019-03079-8

21. Attili I, Tarantino P, Passaro A, Stati V, Curigliano G, de Marinis F. Strategies to overcome resistance to immune checkpoint blockade in lung cancer. Lung Cancer 2021; 154: 151-60. doi: 10.1016/j.lungcan.2021.02.035 